\title{
WORKSHOP ISLAND 3 ALGEBRAIC ASPECTS OF INTEGRABILITY
}

\author{
Islay, Scotland, UK \\ 3-7 July 2007
}

Introduction. As did the very first ISLAND workshop, ISLAND 3 took place on the Hebridean island of Islay, providing a beautiful and serene surrounding for the meeting which ran for over four days. Building on the success of the previous meetings, ISLAND 3 saw the largest number (so far) of participants coming from countries all over the world. A complete list can be found below.

The keynote talks were delivered by international experts in aspects of integrable systems, including Professors Etsuro Date (Osaka, Japan), Boris Dubrovin (SISSA, Italy), Masatoshi Noumi (Kobe, Japan), Simon Ruijsenaars (Leeds, UK), Alexander Varchenko (Chapel-Hill, North-Carolina USA) and Alexander Veselov (Loughborough, UK). These talks reflected the large variety of algebraic aspects featured in integrable systems covering problems of universal critical behaviour of solutions of integrable equations, orthogonal polynomials and discrete integrable systems, interrelation between classical integrable systems and soliton solutions of wave equations, Calogero-Moser systems and Knizhnik-Zamolodchikov equations, the Bethe ansatz method and its application to algebraic geometry. A highlight of the workshop was the talk by Professor John McKay (Concordia, Canada) who pointed out the integrable systems structures appearing in the theory of modular functions. The diversity of the topics covered by the workshop is also reflected in these proceedings, and we wish to thank all the authors for their contributions.

The scientific success and high standard of the conference was matched by the hospitality and excellent food of the Machrie Hotel, Islay, and on behalf of all the local organisers we wish to express our sincere thanks to its staff and the hotel manager.

Well-deserved breaks between talks and scientific discussions were used to sample Islay's famous whiskies and explore its beautiful scenery and its history as the former seat of the Lords of the Isles. We hope that the impressions left by Scotland's hospitality and natural beauty as well as the stimulating scientific discussions held during the conference will see many participants return to the ISLAND workshops to come.

This workshop would have not been possible without the financial support of the following funding bodies: the Daiwa Foundation, the Engineering and Physical Sciences Research Council, the Edinburgh Mathematical Society, the Glasgow Mathematical Journal Trust, the Institute of Physics, the London Mathematical Society, the MISGAM network funded by the European Science Foundation and the Sasakawa Foundation. We gratefully acknowledge all of their financial contributions as well as the one from the Department of Mathematics at the University of Glasgow.

Finally we would like to give our thanks to our international advisory committee,

Harry Braden

Boris Dubrovin

Alexander Veselov
University of Edinburgh

SISSA, Trieste

Loughborough University, 
for its help and suggestions, as well as our co-organisers Chris Athorne, Claire Gilson and Jon Nimmo, the postgraduate students Susan Macfarlane and Craig Sooman, Dr Chunxia Li (visiting on a Royal Society China fellowship) and Mrs Gail Henry from the Department of Mathematics, University of Glasgow, for their help in organising this workshop.

Misha Feigin, Christian Korff and Ian Strachan

The Editors

\section{List of participants}

Christopher Athorne Mariusz Bialecki

Michael Bialy

Harry Braden

Guido Carlet

Oleg Chalykh

Alexander Chervov

Etsuro Date

Yassir Ibrahim Dinar

Adam Doliwa

Boris Dubrovin

Victor Enolski

Misha Feigin

Eugene Ferapontov

Allan Fordy

Claire Gilson

Luc Haine

Martin Hallnäs

Masashi Hamanaka

John Harnad

Jarmo Hietarinta

Luuk Hoevenaars

Nalini Joshi

Kenji Kajiwara

Saburo Kakei

Christian Korff

Chunxia Li

Sarah Lobb

Sara Lombardo

Susan MacFarlane

John McKay

Jun Mada

Kenichi Maruno

Andrey Mironov
University of Glasgow

The University of Tokyo

Institute of Geophysics PAS

Tel-Aviv University

University of Edinburgh

SISSA

University of Leeds

ITEP Moscow

Osaka University

SISSA

University of Warmia and Mazury

SISSA

Institute of Magnetism, Kiev

University of Glasgow

Loughborough University

University of Leeds

University of Glasgow

University of Louvain

Royal Institute of Technology

University of Nagoya

CRM, Montreal

University of Turku

Utrecht University

The University of Sydney

Kyushu University

Rikkyo University

University of Glasgow

University of Glasgow

University of Leeds

Vrije Universiteit Amsterdam

University of Glasgow

Concordia University

The University of Tokyo

University of Texas-Pan American

Sobolev Institute of Mathematics, Novosibirsk 
Folkert Müller-Hoissen Max-Planck-Institute for Dynamics and Self-Organization, Göttingen

Maciej Nieszporski

Jonathan Nimmo

Uniwersytet Warszawski

Masatoshi Noumi

University of Glasgow

Vladimir Novikov

Kobe University

Maxim Pavlov

University of Kent

Andrea Raimondo

Paolo Rossi

Simon Ruijsenaars

Jan Sanders

Cornelia Schiebold

Wolfgang Schief

Alexander Sergeev

Sergey Smirnov

Vladimir Sokolov

P.N. Lebedev Physical Institute, Moscow

Imperial College, London

SISSA

University of Leeds

Vrije Universiteit Amsterdam

Mid Sweden University

Technische Universitaet Berlin

Loughborough University

Moscow State University

Landau Institute for Theoretical Physics

Craig Sooman

Ian Strachan

Tetsuji Tokihiro

Didier Vanderstichelen

Alexander Varchenko

University of Glasgow

University of Glasgow

The University of Tokyo

Université Catholique de Louvain (UCL)

Alexander Veselov

University of North Carolina at Chapel Hill

Claude Viallet

Loughborough University

Centre National de la Recherche Scientifique

Jing Ping Wang

Ralph Willox

Thomas Woodcock

Jun-xiao Zhao

University of Kent, Canterbury

The University of Tokyo

Imperial College London

Service of Physics of l'Etat Condense, CEA France

Dafeng Zuo

Korea Institute for Advanced Study 